\title{
EVALUATION OF A PROPOSED EXPERT SYSTEM DEVELOPMENT METHODOLOGY: TWO CASE STUDIES
}

\author{
Lewey Gilstrap \\ Computer Sciences Corporation \\ 4600 Powder Mill Road \\ Beltsville, MD 20705
}

\begin{abstract}
Two expert system development projects were studied to evaluate a proposed Expert Systems Development Methodology (ESDM). The ESDM was developed for use at Goddard Space Flight Center (GSFC) to provide guidance to managers and technical personnel and serve as a standard in the development of expert systems. It was agreed that the proposed ESDM must be evaluated before it could be adopted; therefore a study was planned for its evaluation. This detailed study is now underway. Before the study began, however, two ongoing projects were selected for a retrospective evaluation. They were the Ranging Equipment Diagnostic Expert System (REDEX) and the Backup Control Mode Analysis and Utility System (BCAUS). Both projects were approximately 1 year into development. Interviews of project personnel were conducted, and the resulting data was used to prepare the retrospective evaluation. Decision models of the two projects were constructed and used to evaluate the completeness and accuracy of key provisions of ESDM. A major conclusion reached from these case studies is that suitability and risk analysis should be required for all AI projects, large and small. Further, the objectives of each stage of development during a project should be selected to reduce the next largest area of risk or uncertainty on the project.
\end{abstract}

\section{INTRODUCTION}

The Expert Systems Development Methodology (ESDM) is intended to be applied to the development of expert systems at the National Aeronautics and Space Administration/Goddard Space Flight Center (NASA/GSFC). The methodology is based on a survey of existing methodologies, experience in developing a number of expert systems at GSFC, and an analysis of the expert system life cycle. Dr. Barry W. Boehm introduced a risk-driven methodology for conventional systems development in his spiral model for software development (Boehm, 1988). ESDM, while independently generated, is also a risk-driven methodology that can be represented by a spiral model with the focus on knowledge acquisition as opposed to product development. Figure 1 shows the spiral model of ESDM.

Risks are inherent in all system development projects, but they are greater in ES development because of the uncertainties associated with modeling human expert decision processes. At the outset of the development of an expert system, it is not known whether an expert's decision processes are cognitive processes that can be modeled by ES techniques. Some human decisions are made on the basis of intuition or skills, which usually cannot be modeled using ES techniques. Intuitive processes and skills can often be modeled using other techniques, such as neural networks, but ESDM does not address these. Even after it has been determined that an expert's decision processes can be modeled, there remain developmental risks because of uncertainties about the robustness and performance that can be obtained from the expert system.

ESDM was developed as a tool for both project managers and developers of expert systems in the NASA environment. It focuses on the knowledge acquisition task, rather than on product development. Key features and recommendations of ESDM include:

- Decomposition of an ES development project into stages. In each stage, work is directed toward the acquisition of the key knowledge needed to reduce the most immediate or highest level risk of the project.

- Explicit identification of the objectives of each stage of work prior to its initiation and testing to verify that the objectives have been met.

- Well-defined criteria for stopping ES development. Once the functional requirements of the proposed system have been identified, ESDM recommends dropping the ES approach and continuing the project along the lines of conventional system development. ESDM also recommends stopping the ES project if the expert's decision processes are not suitable for ES modeling or if an 


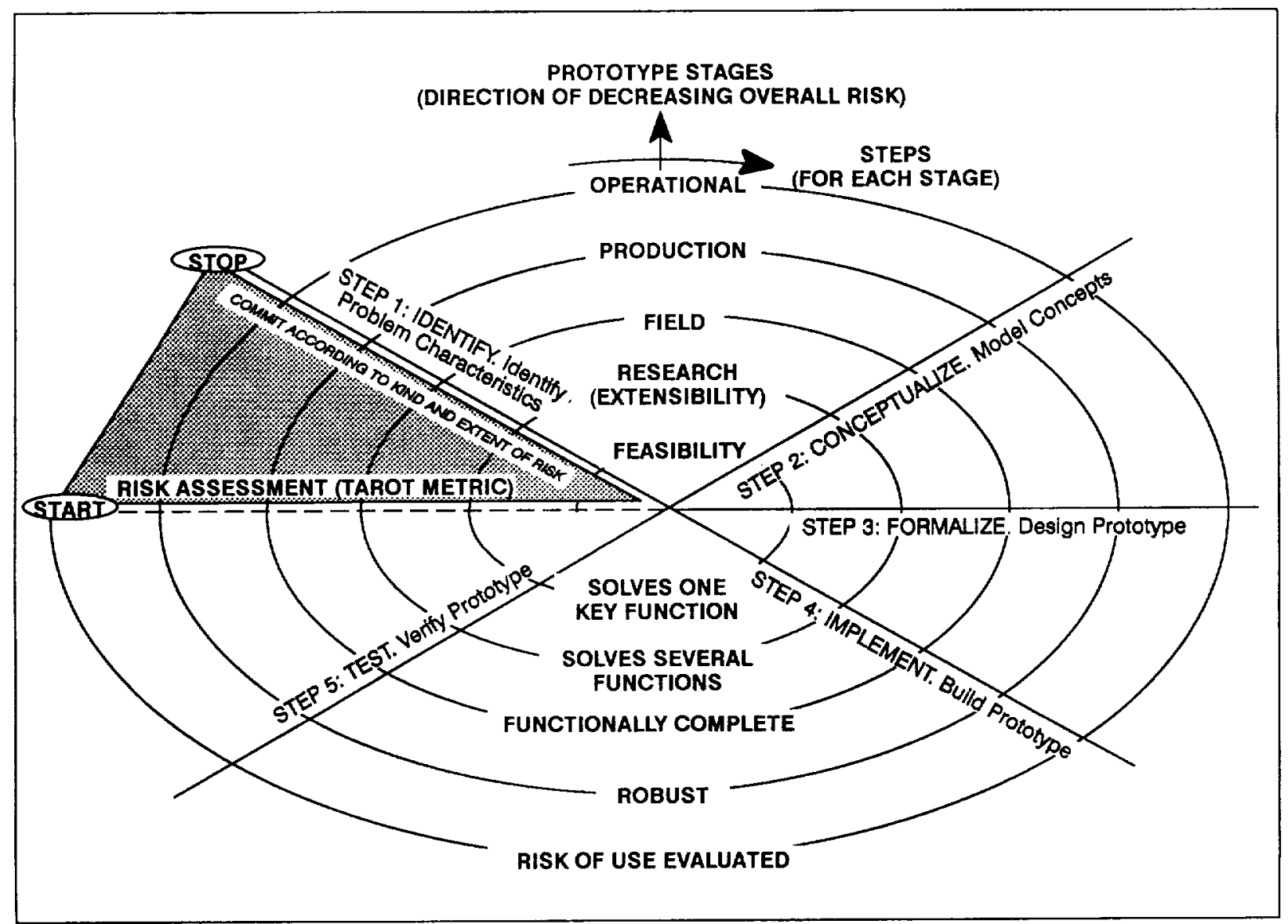

Figure 1. Spiral Model of ESDM

algorithm is discovered that performs the decision process satisfactorily.

- ESDM provides guidance for the kinds of special documentation needed for ES projects and the management information that should be collected for administrative reporting.

- ESDM recommends using of quantitative methods for assessing risk where possible and provides a tool, the Test for Application of Risk-Oriented Technology (TAROT), to assist in this evaluation.

The ESDM project has produced a user's guide (CSCa, 1988), a policy document (CSCb, 1988), and a reference manual (CSCc, 1988) along with training materials. ESDM has been proposed for use on all GSFC expert system projects. Because a proposed methodology must be evaluated before its adoption, however, a framework for the evaluation was also developed (CSC, 1989). The framework recommended selecting an expert system development project and using it as a pilot to evaluate the features of the ESDM before its adoption as a standard. The project would be followed from beginning to end and would collect data on ESDM effectiveness.

Before undertaking a full-scale pilot study, two ongoing projects were selected for a retrospective evaluation of ESDM. The Ranging Equipment Diagnostic Expert System (REDEX) and the Backup Control Mode Analysis and Utility Systems (BCAUS) were the two projects selected. Data on the two projects was collected by interviewing project personnel. Decision models of the two projects were also constructed and used to evaluate the completeness and accuracy of ESDM in accordance with the general provisions of the framework for evaluation.

This paper presents a summary of the findings made on these two case studies. The case studies include a description of the two projects, the key decisions made on the projects, and conclusions reached about the methodology. 


\section{THE REDEX SYSTEM}

REDEX is an advanced prototype expert system that diagnoses hardware failures in the ranging equipment (RE) at NASA's Ground Network tracking stations (Luczak, 1989). REDEX is intended for use by RE technicians in identifying faulty circuit cards or modules that must be replaced. The system has a highly graphical user interface that uses color block and layout diagrams to illustrate fault locations. Figure 2 shows the environment for REDEX.

The REDEX project was initiated by the Telecommunication Systems Branch (Code 531) at GSFC as a task assignment. There were two persons assigned to the project initially, but the level of effort has averaged less than two full-time persons.

No formal risk or suitability analysis of the project was performed. The use of an expert system as a diagnostic aid for the RE was considered feasible because the RE had been designed with a large number of built-in test points. It was expected that these test points would greatly facilitate the automation of fault diagnosis, and the task of REDEX was to speed up the identification process.

Development staff personnel were generally familiar with the provisions of ESDM. On their own initiative, they selected ESDM features that they believed would assist them in the development of REDEX and used them in the project. The selected features were:

- The use of a staged development

- The decomposition of stages into steps
- The use of risk analysis to guide the selection of objectives for stages

The stages of work on REDEX followed ESDM recommendations closely for addressing successively more complex objectives. Five stages of work were defined, each addressing more complex issues. The following summarizes these five stages:

1. Feasibility of implementing one diagnostic rule and accomplishing diagnosis with this rule

2. Feasibility of extending the feasibility prototype to include all relevant rules on the selected hardware host (IBM PC-AT)

3. Feasibility of implementing one graphics screen on the selected host

4. Feasibility of extending the graphics system to include all required graphics

5. The capability' of the system to be fielded (field prototype), including handling all necessary communications with the equipment

REDEX is implemented in Prolog on an IBM PC AT-compatible workstation. A semantic network knowledge representation technique was used to model the design structure of the RE. A catalog of generic troubleshooting rules was compiled to represent heuristics that are applied in diagnosis. Specific troubleshooting rules unique to the RE were also added. Over 50 generic and 250 specific rules were developed. A hypertext-like scheme is used to allow the user to navigate through the diagrams and tables. Over 50 graphic and tabular displays have been implemented.

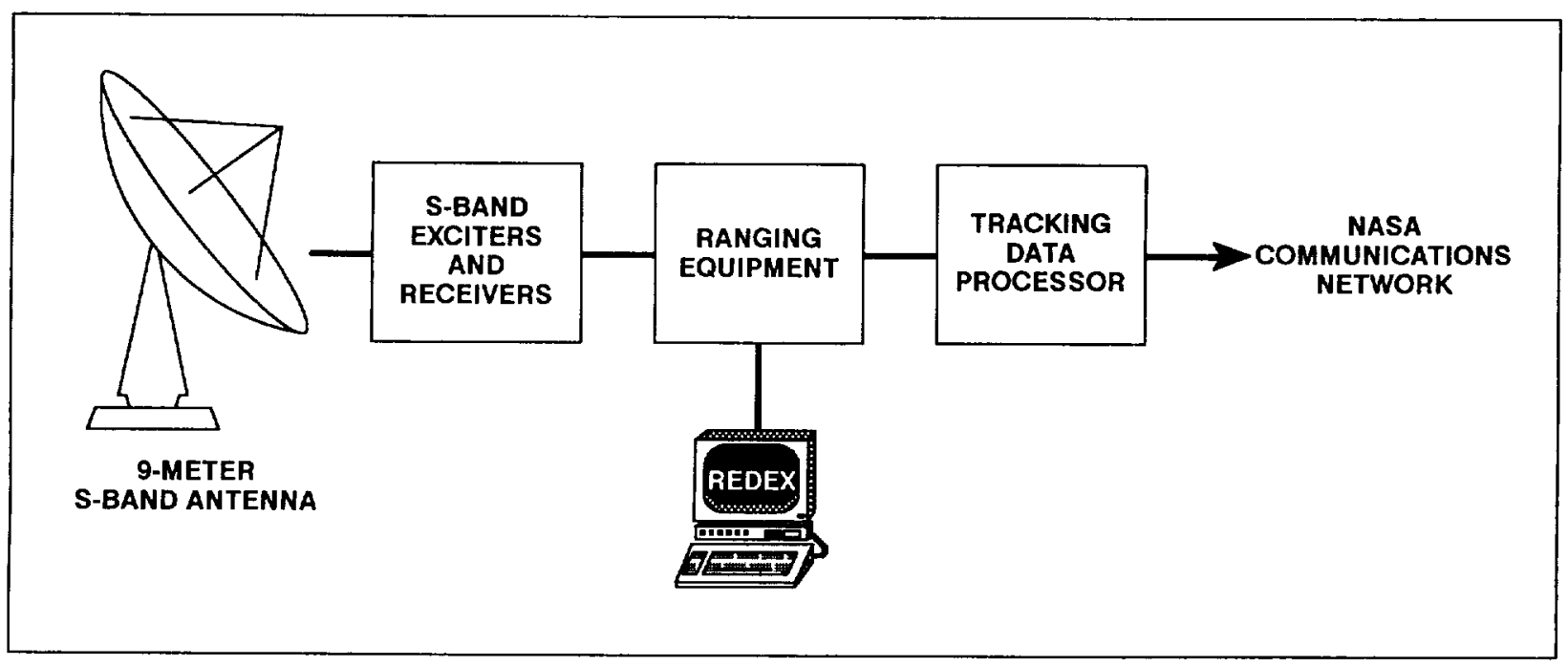

Figure 2. NASA Ground Network Tracking Station 
The client identified the use of IBM PC-AT hardware as desirable at the outset of the project. The choice of computer language or shell development system was left to project personnel, but an early identification of the language was requested for budgetary estimation. At project initiation, it was not clear what knowledge representation scheme might be most appropriate for the knowledge yet to be acquired, and project personnel began addressing this problem immediately. After investigation, they determined that a rules representation would be appropriate. Generic and specific rules (in a pseudocode form) for the system were manually compiled.

From the somewhat limited choices available for the implementation of knowledge-based systems on an IBM PC-AT, project personnel concluded, after further investigation of six languages and shell development systems, that Prolog appeared to be suitable for prototyping the rules. A feasibility prototype was then developed to test whether Prolog was suitable for the system prototypes. (Prolog's suitability for the operational system was not determined at this time; however, its suitability in this regard has since been proved.) At the completion of the second prototype stage, called the research stage in ESDM, the functional requirements for REDEX were validated. These requirements were then documented and issued in a functional requirements document.

The fifth (and current) prototype stage of REDEX addresses those risks associated with using the system in the field. In this prototype, the uncertainties are concerned with the communications between the RE and REDEX. This system is currently being evaluated in a communications emulation testbed and will be connected to the RE after evaluation.

\section{THE BCAUS SYSTEM}

BCAUS is an expert system designed to assist flight operations personnel in diagnosing the cause of a Gamma Ray Observatory (GRO) spacecraft autonomous mode transition (Bush, 1989). The GRO spacecraft was designed with onboard capability to safe itself autonomously, transitioning from a primary operating mode to a backup control (safing) mode in the event of certain error conditions in the attitude control and determination (ACAD) subsystem.

Flight operations personnel need to understand what error condition trigger the onboard computer (OBC) to order the mode transition and why that error condition occurred so that they may take the proper corrective action. The $O B C$ was not designed, however, to provide the triggering information or the diagnostic information to the operator.
Input information to BCAUS will be provided by telemetry data from GRO and by user input. Output from BCAUS will be provided only to the diagnostician. There is no output back to the spacecraft. Figure 3 shows a diagram of the information flows in BCAUS.

GSFC also initiated the BCAUS project by issuing a task assignment. Two persons were assigned to the project. No formal risk or suitability analysis of BCAUS was performed. Task personnel had knowledge of the risk areas in expert system development and used this information to guide the development process. The primary area of risk for BCAUS was in the knowledge acquisition process. Four sources of expertise were identified and were initially considered adequate for the development task. These four sources were (1) documentation, (2) GSFC spacecraft design experts, (3) GSFC flight operations experts, and (4) TRW personnel associated with the design of the relevant GRO subsystems. However, project personnel found that the knowledge acquisition task for this system was more difficult than initially thought and that the initial evaluation of risk had to be modified. The project goals have therefore shifted from providing an operational system to a system in which the knowledge base is easily modified and updated on the basis of actual experience. In brief, the goal has shifted from providing an initially operational system to an adaptive system with an initial base of knowledge that can be upgraded as expertise is acquired.

The difficulty in the knowledge acquisition task for the BCAUS project is that the expertise needed to diagnose GRO mode transitions has not yet been acquired by humans. There was no training course available for GRO fault diagnosis as there was for REDEX. The existence of a training course means that the diagnostic knowledge has been compiled, thus implying a lower risk of system development. However, even the designers of the GRO subsystems had not yet acquired or compiled all the information necessary for mode transition analysis, and this fact was not known at the outset of the project. The relative inaccessibility of the TRW design engineers because of their location on the west coast and their limited availability for consultation made it difficult for project personnel to elicit any available information. When the full difficulty of knowledge acquisition became known, a reevaluation and reorientation of project goals and objectives became necessary. The complexity of the knowledge acquisition task on the BCAUS project perhaps doubled the time required to reach a feasibility prototype. This situation constrained the design of the operational system in ways 


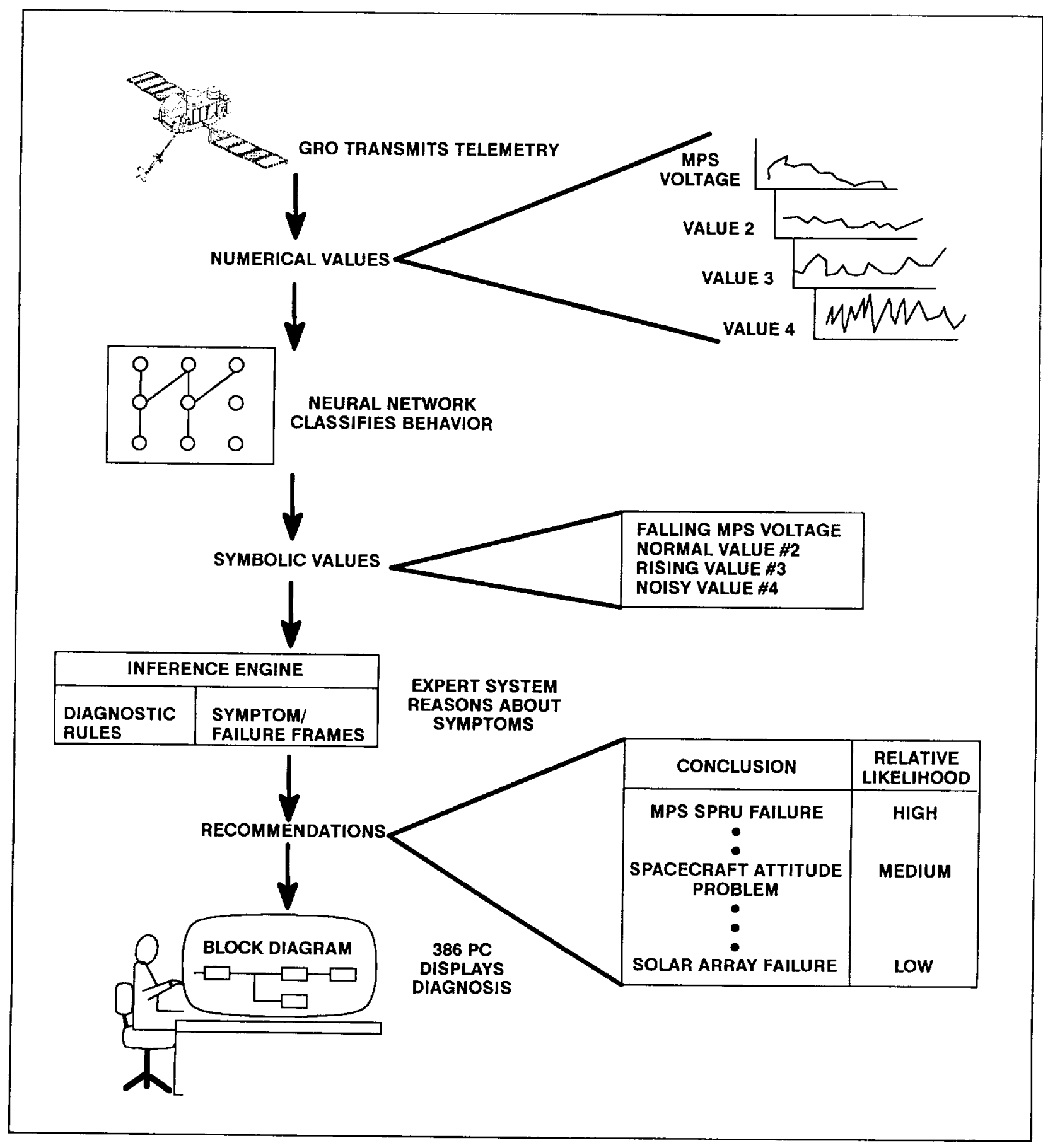

Figure 3. The BCAUS System

that were not and probably could not have been, determined at the outset.

The first prototype system, a feasibility prototype, was implemented on a PC-386 class machine using the KES hypothesize-and-test (HT) inference engine developed by Software Architecture and Engineering, Inc. Basic structural knowledge of the system elements was loaded on the machine in three weeks by two persons. No rules were needed because of the built-in diagnostic feature of KES HT. When KES HT was selected initially, there were some 
known limitations on its capabilities. Following its use on the project, it became clear that the limitations were too restrictive, especially in the area of explanatory power. The second prototyping system selected was ART-IM, an expert system development shell produced by Inference Corporation, which can run on both the 386 machine and a Silicon Graphics Iris $4 \mathrm{D} / 20$. The Iris has considerably more power for graphics than the 386 machine.

The BCAUS system will also have a neural network front end to provide trending data on input telemetry signals. Project personnel determined that it might be possible to implement trending analysis using ART-IM rules, but at the expense of making the system much larger and more complex than desirable. The software product, Neural Works Professional II, from Neural Ware, Inc., was selected to implement the trending analysis.

The BCAUS system's graphics interface shows relevant subsystems in the form of functional block diagrams, similar to those implemented in REDEX, with highlighted potential problem areas. A hierarchical traverse is planned for navigation of the diagrams and causal graphs.

As was the case with REDEX, there was a strong tendency to follow the methodology used for conventional software systems, and the hardware and software selections were set very early in the project. The deadlines for hardware and software selection were met only through very concentrated effort on the part of the project's development staff.

\section{PROJECT KEY DECISIONS}

In the evaluation process, ESDM was modeled as a sequence of key decisions plus subsidiary decisions. Key decisions are identified on the basis of their possible impacts on project cost and schedule. The key decisions of ESDM are:

- Start. The decision that the project is suitable for implementation by an expert system is based on a formal suitability analysis in ESDM.

- Knowledge-oriented approach. Is current knowledge about the problem sufficient to permit preparation of specifications for the system now? If not, then a knowledgeoriented approach is indicated, that is, the decision is made to acquire the missing information first.
- Staffing. ESDM recommends a knowledge engineer, AI programmers, system programmers, and domain experts for expert system projects.

- Staging. ESDM recommends dividing a project into successive stages based on the degree of uncertainty about how to accomplish the function or service.

- Steps within stages. ESDM recommends following five steps within each stage. These steps focus on identifying the knowledge to be acquired within the stage, on acquiring this knowledge, and on verifying the correctness of the acquired knowledge.

- Explicit risk evaluation. ESDM recommends that all risk evaluation be explicit, that is, that each area of risk on the project be documented and assessed. ESDM also provides a formal tool, the TAROT metric, to assist in estimating of risks.

- Stop-rule. This decision is based on acquiring sufficient information to prepare meaningful and realizable specifications. At this point, ESDM recommends continuing the project as a normal software development project following the conventional software development life cycle.

There are also technical and managerial decisions of lesser importance that have some bearing on project schedule and costs:

- Reporting (frequency, type, content)

- Hardware and software tools selection and timing

- Use of automated knowledge tools

- Need for graphics and interfaces

The method used to evaluate ESDM was, first, rating how closely the circumstances and decisions of the two projects matched the provisions of ESDM and, second, assessing the worth of the provisions based on the experience gained on the projects.

The start decision on both REDEX and BCAUS, that is, the decision to use an expert system or knowledgebased technology to develop a system was made by GSFC personnel, not by the development project personnel. There is no information on whether any formal analysis of suitability was made by GSFC personnel. In retrospect, it is clear that both projects were, in fact, suitable. By now, the usefulness of expert systems for fault diagnosis has been well established; this fact can be considered generally well known in the computer field.

The ESDM provision that recommends a suitability analysis for each new project should be amended to 
take into account current practices, informal standards, and common knowledge among practitioners in the computer field. The study concluded that a more formal analysis of suitability should still be performed for any system that does not fall into one of the familiar categories of expert system applications. The decision regarding a formal suitability analysis on any new project is a judgment call. Nevertheless, ESDM must continued to provide the guidelines and procedures for cases requiring a suitability analysis.

It was apparent even from a casual analysis of both REDEX and BCAUS that it was impossible to prepare specifications at the outset for either project and that a knowledge acquisition process would be required. What is important, however, is that knowledge acquisition procedures are required and that identifying the missing pieces of information is necessary in order to develop the systems. The identification of this information was carried out on both projects.

Both the REDEX and BECAUS projects were staffed with experienced AI professionals. ESDM guidelines call for both knowledge engineers and $\mathrm{AI}$ programmers. Because of the small size of the projects, however, it was necessary for project personnel to function both as knowledge engineers and as AI programmers. Also, project personnel assumed some of the functions of domain experts ESDM should be modified to take the special requirements of small projects into account, but the need for experienced and competent staff personnel becomes even more acute for these smaller projects. Managers should remain aware of the staffing requirement differences in small and large projects.

Both REDEX and BCAUS were decomposed into successive stages of work. ESDM recommends defining stages in terms of risk and addressing areas of highest risk first. While there was no conscious decision to follow ESDM provisions on selecting stages, REDEX personnel nevertheless followed the feasibility, research, and field stages quite faithfully. REDEX also decomposed the planned system into three subsystems (functional, user interface, and communications interface) and followed the riskreduction sequencing in each subsystem. Staging was also followed on BCAUS. The first year of work on BCAUS was considered to be the feasibility stage.

ESDM defines the research stage as that stage of work that establishes that one or a small set of rules can be implemented. The issue to be addressed then is whether enough of the required rules can be implemented to make the system practical. A better name for this stage should reflect the intent of the stage, that is, determining how far the feasibility prototype can be extended. The name, extensibility stage, has been suggested as a replacement.

Both projects followed some natural sequence of work within the stages that was similar to the steps described in ESDM. In fact, the steps within the stages recommended in ESDM are a paraphrase of the scientific method, which is the model for knowledge acquisition or discovery processes.

There was no formal analysis of risks made on either REDEX or BCAUS; however, both development teams reported being acutely aware of the risks associated with different areas of their projects at all times and stated that their work was governed by this awareness. This awareness of risk characterizes the experience of the development teams. Less experienced personnel might not have the opportunity to put together workable and useful systems.

On small projects, there is less need for formal analysis of risk. The lack of a formal analysis on small projects should not be a concern to managers, as long as the staff is aware of risks and is guided by their consideration. On large projects, the use of a formal risk analysis is still recommended. ESDM provisions are being modified to take the size of the project into account.

There are no plans to transfer REDEX or BCAUS to a conventional development cycle after preparation of system requirements. On small NASA projects, the personnel who began the project will typically carry on the development even after requirements have been specified and risks reduced to an acceptable levels. Transfer to a conventional life cycle with a new development team, which was recommended in ESDM for large projects, will probably be the exception, rather than the rule, for most small projects.

The documentation prepared on the two projects tended to follow the requirements for conventional software development. Although it is impossible to draw conclusions about ESDM provisions for documentation, project personnel felt that the knowledge acquisition process was not adequately documented by the normal system development documents, thus lending support to the ESDM provisions. Some adjustment for the number of documents recommended by ESDM should be made on the basis of project size.

Similar to the typical requirements associated with conventional software engineering, the hardware and software tools to be used on a project must be specified at the outset or as early as possible. Based on the two retrospective studies, the conclusion is that 
ESDM provisions are the least risky, that is, delaying the selection of hardware and software tools until after identification of the knowledge structure.

Because of the experiences gained by project personnel on REDEX and BCAUS, they suggested the possibility of using automated tools for logging and managing lists of rules or other knowledge structures. Since the final evaluation is incomplete at this time, therc has been no change in ESDM regarding recommendations on the use of automated tools.

In addition to the two projects described in this study, there was a review of a number of other expert systems prepared for NASA. Nearly all of them made use of color graphics interfaces for presentation of information to the users. This fact has some implications for the selection of tools for the development of expert systems and the selection of personnel to work on expert systems. A possible modification to ESDM will point this out and provide guidelines for tool and personnel selection.

REDEX makes use of input data from equipment monitoring points and BCAUS has telemetry inputs from the GRO spacecraft. Many NASA expert systems have sources of input information other than the human. Also, many make use of other techniques than logic programming, such as:

- Neural networks

- Procedural code

- Operating system calls

The staffing requirements provisions of ESDM that address only knowledge engineering and AI programming should be modified to take into account the possible needs for systems programming, neural net programming, and familiarity with telemetry and communications.

\section{CONCLUSIONS}

The two projects surveyed match the model of the expert system development life cycle so closely that the experience gained on these projects provides valuable information for ESDM evaluation. The two projects are quite different in detail and dynamics, and they differ from the expected large-size project envisioned by ESDM. The experience of these projects is useful primarily in providing ESDM with extensions to cover the cases of small-size projects.
General conclusions reached from the retrospective study of the two projects include:

- Confirmation of the need for a methodology. The standard systems development methodology matches the life cycle of expert systems poorly. The need for a methodology better suited to the special requirements of expert systems is supported by project experience.

- Support for the use of a risk-based approach. Both project teams reported that they were aware of risks in development and organized their projects to address these risks. ESDM formalizes this practice in ES development.

- The decomposition of projects into successive stages. Both projects broke the work down into successive stages to make the overall task more manageable.

- Requirements as an overall goal. Both projects produced requirements documents at the conclusion of an extensive knowledge acquisition phase in accordance with the recommendations of ESDM.

Based on the evaluations provided by the two projects, REDEX and BCAUS, it was possible to reach some specific conclusions about the details of ESDM and the framework to be used for its evaluation on the two pilot projects.

- ESDM currently requires a formal suitability analysis for all projects. Findings suggest that this requirement should be relaxed for small projects.

- ESDM should be modified to describe the differences between small and large projects. In particular, some of the formal documents required for projects are unnecessary for small projects and may impose an unnecessary burden.

- The name of the research stage of the ESDM life cycle should be changed to the extensibility stage.

- The use of the TAROT metric (or other formal tool) for evaluation of risk and the suitability of candidate projects for ESDM should be optional for small projects.

- Personnel qualifications for expert systems development should include experience and familiarity with graphics user interfaces as well as with the functional tools required for expert systems. 


\section{REFERENCES}

Boehm, B. (May 1988). A spiral model of software development. IEEE Computer.

Bush, J. L. and Weaver, S. J. (May 1989). BCAUS project description and consideration of separation of data and control. NASA Conference Publication 3033.

Computer Sciences Corporation (a) (September 1988). Expert system development methodology (user's guide), DSTL-90-004.
-- (b) (September 1988). Expert system development methodology (policy document), DSTL-90-005.

- (c) (September 1988). Expert system development methodology (reference manual), DSTL-90-006.

-- (March 1989). Expert system development methodology; Framework for evaluation of ESDM.

Luczak, E. C., Gopalakrishnan, K., and Zillig, D. (May 1989). REDEX: The ranging equipment diagnostic expert system. NASA Conference Publication 3033. 
